\title{
Study of the measurements placement problem for planning of the optimal experiment
}

\author{
Oksana A. Grebneva ${ }^{1, *}$ \\ ${ }^{1}$ Melentiev Energy Systems Institute of Siberian branch of Russian Academy of Science (ESI SB \\ RAS), department of pipeline and hydraulic systems, Lermontov street 130, Irkutsk, Russia, 664033
}

\begin{abstract}
Both in Russia and abroad, there are works that are devoted to the problem of optimal placement of measuring devices, which are evidenced by the current literature. The proposed methods are not universal, that does not allow them to be directly used for different types of pipeline systems. In addition, the developed algorithms does not guarantee a global solution. In this regard, there is a demand for solving the problem of optimal placement of measuring devices for pipeline systems. At the same time, not only the number and accuracy of measuring devices, but also their composition and placement locations are important. In this paper, a mathematical formulation of the problem of optimal placement of measuring devices is given, methods for its solution are proposed. The numerical example shows the effectiveness of the proposed method of optimal placement of measuring devices, which allows to get a global solution for a previously known finite number of steps.
\end{abstract}

\section{Introduction}

Recently, due to new trends in the transition to intelligent pipeline systems (PLS), technological transformation and modernization of PLS is taking place through the introduction of new equipment, including measuring devices, measurement data collection and processing systems, etc., on the one hand, development of computer technology, methods of mathematical modeling, on the other. Despite the equipment of PLS with measuring devices, which, as a rule, are installed at the main PLS facilities (sources, pumping stations, etc.) for technological and commercial account; this is not always enough to ensure the identifiability of the PLS, which means the possibility of recovery mathematical models on the results of measurements and observations of their functioning. The lack of adequate models is a major deterrent to the effective application of mathematical and computer modeling methods.

Significant results in the area of identification problems for PLS were obtained in Russia [1-3]. Recently, the problem of identification has received considerable attention abroad [4-11]. Works in this area are called «model calibration» tasks. On the one hand, the use of most of proposed methods allows significantly reduce the number of uncertain parameters, on the other hand, by averaging the initial information, reduces the adequacy of

*Corresponding author: oksana@isem.irk.ru 
models obtained with model calibration. In addition, in these works the problem of observability and identifiability of the PLS, as the fundamental cybernetic properties, is not considered.

The problem of optimal placement of measuring instruments is considered in works both in Russia and abroad. The current literature indicates this [12-20]. The object of application for the developed methods and algorithms are electric power systems [18-20] and water supply systems [12-17]. The proposed methods are not universal, that does not allow them to be directly used for different types of PLS. In addition, the developed algorithms do not guarantee a global solution.

In this connection, the need for solving the problem of optimal placement of measuring devices for a PLS arises. At the same time, not only the number and accuracy of measuring devices, but also their composition and placement locations are important.

In ESI SB RAS, within the framework of the new approach to identify of PLS [1,21], an approach was proposed for optimal placement of measuring devices [22,23]. In this paper, studies on the possibility of applying the developed approach to solving the problem of measuring devices placement for ensure of PLS identifiability are given.

\section{Mathematical statement of the problem}

Let the PLS operation state and some redundant composition of measurements be set. In this case, the task of the optimal measurements placement is to minimize the number of measuring devices [23] $\left(\min \sum_{j=1}^{l} \delta_{j}{ }^{(u)}\right)$ subject to their number $q \leq \sum_{j=1}^{l} \delta_{j}^{(u)} \leq \bar{l}$ and a given concession in the optimality of the information criterion $\operatorname{det} C_{X}$. There $l$ is the number of measurable variables; $\delta^{(u)}$ is an $r$-dimensional vector of Boolean variables in the $u$-th state with components: $\delta_{j}^{(u)}=1$, if the $j$-th variable is measured in the $u$-th state and $\delta_{j}^{(u)}=0$, otherwise, $j=1, \ldots, r ; \bar{l}$ is the number of variables from a set of components of vector $R$ that are accessible for measurements; $q$ is the minimum necessary number of measurements.

To solve of this problem it is proposed to use the determinant of the covariance matrix, known in the experiment planning theory as the D-criterion [24-27], which is an estimate of the degree of uncertainty of parameter vector as a whole. Therefore, the smaller the value of the determinant, the less this uncertainty.

Also an important point is the equivalence property $D$ - and $G$-optimal plans of experiment $[24,25]$, according to which, the optimal plan will simultaneously provide the best predictive properties of the identified model, thus the optimal value of the $D$-criterion will correspond to the minimum of the maximum variance of dependent state parameters.

However, the $D$-criterion makes it possible to know about the degree of observability and parametric identifiability (higher or lower), but it does not allow evaluating quality of the parameter estimation, i.e. value of their accuracy. Therefore, as an additional criterion for solving the problem, we use the criterion $\gamma=\max _{j} \frac{\hat{\sigma}_{j}^{2}}{\tilde{\sigma}_{j}^{2}}$, where $\hat{\sigma}_{j}^{2}$ is calculated (a posteriori) variance of the non-measurable parameter of the state $j ; \tilde{\sigma}_{j}^{2}$ is known (prior) variance of the device error for measuring this parameter. The value $\gamma$ allows estimating accuracy of determining the non-measured parameter and shows what fold the variance of the estimate of the non-measured parameter is greater than the variance of its direct measurement. 


\section{Methodical approach}

In [28] it was shown, that

$$
\operatorname{det}[\mathbf{F}(l+1)]=\operatorname{det}[\mathbf{F}(l)]\left(1+\hat{\sigma}_{l+1}^{2} / \tilde{\sigma}_{l+1}^{2}\right)
$$

Expression (1) illustrates the relationship between the $D$-criterion and the criterion $\gamma$ and shows that the maximum increase in the criterion $\operatorname{det}[\mathbf{F}(l+1)]$ can be achieved by adding a measurement of the parameter from the vector $Z_{2}$ for which the ratio of the predicted variance to the variance of the measuring is greatest. It is important to note that this parameter can be both dependent and independent parameter of the model.

Respectively

$$
\operatorname{det}[\mathbf{F}(l-1)]=\operatorname{det}[\mathbf{F}(l)]\left(1-\hat{\sigma}_{i}^{2} / \tilde{\sigma}_{i}^{2}\right),
$$

where $\hat{\sigma}_{i}^{2}=\bar{J}_{i}[F(l)]^{-1} \bar{J}_{i}^{T}$. Thus, a decrease in the determinant of the information matrix will be minimal when a measurement is deleted with a minimum value $\hat{\sigma}_{i}^{2} / \tilde{\sigma}_{i}^{2}$, i.e. the minimum value.

Obviously, maximum accuracy can be achieved with the maximum permissible number of measurements измерений $l_{\max } \leq \operatorname{dim}(\mathbf{Z})=r$. The minimum number of measurements is equal to the number of independent parameters $l_{\min }=\operatorname{dim}(\mathbf{X})=q$.

When using the covariance matrix of independent parameters as an information criterion, relation (5) will take the following form

$$
\operatorname{det}\left(\mathbf{C}_{\alpha}(l-1)\right)=\operatorname{det}\left(\mathbf{C}_{\alpha}(l)\right) k_{i},
$$

where $k_{i}$ is the coefficient that allows to find the determinant of the covariance matrix of the elements parameters when one measerment is removed from the measurement vector.

Based on the use of expressions (2) and (3), an efficient algorithm for step-by-step reduction of the measurements number was proposed, at each step of which the admissibility of the increase in the criterion $\operatorname{det} C_{X}$ is checked:

1) the maximum possible nondegenerate composition of measurement is given $\bar{l}$;

2) calculated $\operatorname{det} C_{X}$ and prediction variances for each model parameter;

3) for each of the parameters it is calculated the value of the coefficient $k_{i}$. It is tracked index $i$ for $k_{i \min }$. The corresponding measurement is removed from the current composition;

4) the obtained composition is checked for degeneracy. Then the number of measurements is taken smaller by one and the calculation is repeated from paragraph 2 to 4 until the condition on the minimum permissible number of measurements or on the achievement of the minimum acceptable level of estimation accuracy by the criterion $\gamma$.

The essence of the proposed technique is to consistently reduce the maximum allowable composition of measerments with the exception of the measurement that would minimally worsen the information criterion. This technique allows you to get a global solution in a finite limited number of steps. 


\section{Numerical examples}

For clarity, we will demonstrate the solution to the measurement placement problem for planning of the optimal experiment on the example of a nominal PLS presented in Figure 1.

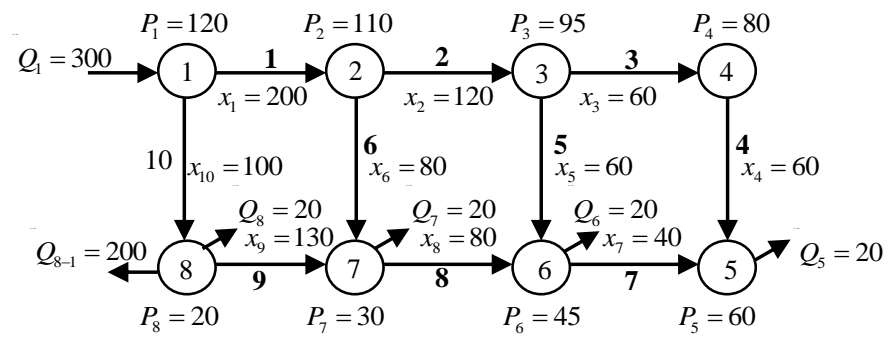

Fig. 1. The scheme of a nominal PLS with initial values of independent parameters $\left(P_{j}\right.$ is the pressure in node $j$ (m.w.c); $x_{\mathrm{i}}$ is flow rates on the branch $i\left(\mathrm{~m}^{3} / \mathrm{h}\right) ; Q_{j}$ is discharge in node $j\left(\mathrm{~m}^{3} / \mathrm{h}\right) ; s_{i}$ is hydraulic resistance on the branch $\left.i\left(\mathrm{u}^{2} / \mathrm{M}^{5}\right)\right)$

The model of steady-state hydraulic regime is used as the initial model [29]. Number of model equetions $\operatorname{dim}(\mathrm{U})=m+n$, where $m$ is number of nodes, $m=8 ; n$ is number of branches, $n=10$. Consequently $\operatorname{dim}(\mathrm{U})=18$. The dimension of the model parameters vector and the number of possible measurements $r=l=\operatorname{dim}(\mathbf{Z})=2(m+n)=36$. The maximum possible number of measurements $\bar{l}=r=36$. The minimum permissible number of measured parameters from the solvability condition of the problem $l_{\text {min }}=q=\operatorname{dim}(\mathbf{Z})-\operatorname{dim}(\mathrm{U})=18$.

Initial information about network: steady flow in the hydraulic circuit is described by twelve hydraulic parameters, including nodal pressures and flow rates $\left(P_{j}, Q_{j}, j=1,8\right)$, as well as hydraulic resistances and flow rates in branches $\left(s_{i}, x_{i}, i=1,10\right)$. For the parameters that can be measured, the following values of standard deviations were accepted: for pressures $\sigma_{p_{j}}=0,2$; for nodal flow rates and flow rates in branches $\sigma_{Q j}=0,5$ and $\sigma_{x i}=0,5$, respectively; hydraulic resistances in branches were supposed to be known with accuracy $\sigma_{s i}=0,0001$.

Initially, the calculation was carried out by the method of full enumeration. In result of the calculation, a range of determinant values $\operatorname{det} \mathbf{C}_{X}$ wit deterrent $\mathrm{h}$ the boundary maximum and minimum values was obtained (Figure $2 \mathrm{a}$ ). This calculation was carried out

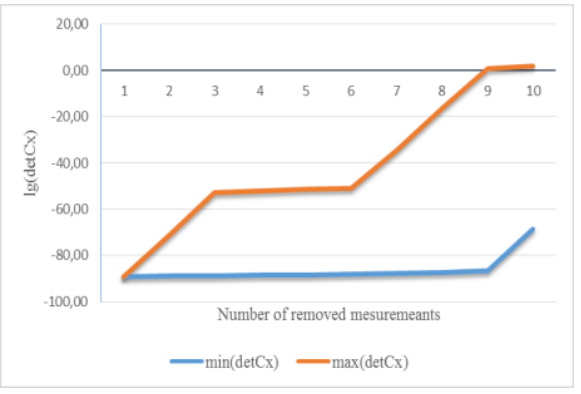

a)

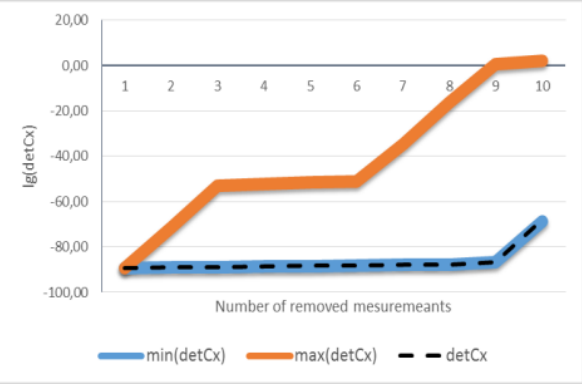

b)

Fig. 2. a) A range of value $\operatorname{det} \mathbf{C}_{X}$; b) Illustration of the operation of the sequential reduction algorithm of measuring devices 
in order to determine the actual values of the objective function for variants of the measurement placement after preliminary screening with the aim of their subsequent comparison with the results of applying the proposed method.

The results of problem solving with the use of a sequential reduction algorithm for measuring devices are presented in Figure $2 \mathrm{~b}$ and in table 1.

Table 1. The process of finding of the optimal placement of measuring devices in the planned state

\begin{tabular}{|c|c|c|c|c|c|c|c|c|}
\hline 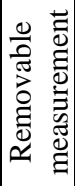 & $\lg \left(\operatorname{det} C_{x}\right)$ & $\gamma$ & 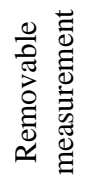 & $\lg \left(\operatorname{det} C_{x}\right)$ & $\gamma$ & 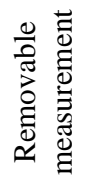 & $\lg \left(\operatorname{det} C_{x}\right)$ & $\gamma$ \\
\hline \multicolumn{9}{|c|}{$\begin{array}{l}\text { The initial composition of the measurement vector } \\
5, P_{6}, P_{7}, P_{8}, Q_{1}, Q_{5}, Q_{6}, Q_{7}, Q_{8}, x_{1}, x_{2}, x_{3}, x_{4}, x_{5}, x_{6}, x_{8}, x_{9}, x_{10}\end{array}$} \\
\hline \multicolumn{9}{|c|}{$-41,2$} \\
\hline$x_{10}$ & $-88,96$ & 0,9999 & $x_{8}$ & $-88,44$ & 1,0178 & $Q_{6}$ & $-87,66$ & 1,1999 \\
\hline$x_{4}$ & $-88,79$ & 0,9999 & $x_{3}$ & $-88,14$ & 1,0179 & $x_{9}$ & $-86,87$ & 1,2117 \\
\hline$x_{2}$ & $-88,63$ & 1,0188 & $x_{1}$ & $-87,83$ & 1,1179 & $P_{4}$ & $-68,57$ & 2,2198 \\
\hline \multicolumn{9}{|c|}{$P_{1}, P_{2}, P_{3}, P_{5}, P_{6}, P_{7}, P_{8}, Q_{1}, Q_{5}, Q_{7}, Q_{8}, x_{5}, x_{6}$} \\
\hline
\end{tabular}

As can be seen, the values obtained with using the proposed algorithm completely coincide with the values of the full enumeration (small deviations in some values are due to the error of calculations). The results of numerous practical calculations confirm that the trajectory of the search for a solution according to the above algorithm corresponds to the lower boundary of the graph (lower line in Fig. 2b). This suggests that the proposed method of optimal measurement placement makes it possible to obtain a global solution in a finite number of steps $K=\bar{l}-l_{\min }$.

\section{Conclusion}

Based on numerical studies, it has been shown that the proposed method for optimal placement of measuring devices allows to stop at any step of solving of the problem and choose a variant of the composition of measuring devices with the required their number and minimal concession in accuracy. In addition, this technique allows getting a global solution for a previously known finite number of steps.

The research was carried out within the project III.17.4.3 of the Fundamental research program of SB RAS (AAAA-A17-117030310437-4) with finance support of RFBR and the Government of Irkutsk Region in the framework of research project № 17-48-380021

\section{References}

1. N.N. Novitsky, Evaluation of hydraulic circuits parameters (Science, Novosibirsk, 1998) [in Russian] 
2. N.N. Novitsky, News of the Russian Academy of Sciences. Energy, 6, 87 (2000) [in Russian]

3. M.G. Sukharev, R.V. Samoilov, Analysis and control of steady and non-steady states of gas transportation (RGU Oil and Gas, Moscow, 2016) [in Russian]

4. S. Lingireddy, L. E. Ormsbee, Civil. Eng. and Env. Syst., 19(1), 13 (2000)

5. Y. Wu. Zheng, T. Walski, R. Mankowski, G. Herrin, R. Gurrieri, M. Tryby, AWWA IMTech Conference (2002)

6. Yi. Wu. Zheng, Journal of Environmental Science and Health, A(41), 1 (2006)

7. Yi. Wu. Zheng, T. M. Walski. International Conference of Computing and Control in the Water Industry, 1 (2005)

8. O. Giustolisi \& E. Todini, Urban Water Journal, 6(1), 39 (2009)

9. B. Xu and A. Abur, IEEE Xplore (2004)

10. T. Fang, R. Lahdelma, Applied Thermal Engineering, 73(1), 1211 (2014)

11. J. Wang, Zh. Zhou, J. Zhao, Energy Conversion and Management, 120, 294 (2016)

12. B.H. Lee and R. A. Deininger, Journal of Environmental Engineering, 118, 1 (1992)

13. A. Ostfeld, J.G. Uber, et al, Journal of Water Resources Planning and Management, 556 (2008)

14. A. Preis and A. Ostfeld, Proc. 8th Annual Water Distribution Systems Analysis Symposium, 1 (2006)

15. M.E. Tryby, M. Propato, and S. Ranjithan, Journal of Water Resources Planning and Management, 1 (2010)

16. P. Auckenthaler and L. Shuming, The 11th International Conference Computing and Control for the Water Industry, 5 (2009)

17. L. Cozzolino, R. D. Morte, A. Palumbo \& D. Pianese, Civil Engineering and Environmental Systems, 28 (1), 75 (2011)

18. A.Z. Gamm, I.I. Golub et al., Assessment of the Condition in the Electric Power Industry (SEI, Irkutsk, 1983) [in Russian]

19. A.Z. Gamm, I.I. Golub, Observability of electric power systems (Science, Moscow, 1990) [in Russian]

20. B. Xu, A. Abur, IEEE PES (2004)

21. N.N. Novitsky, O.A. Grebneva, Energy Pipeline Systems: Models, Applications, Information Technologies, 90 (2000) [in Russian]

22. N.N. Novitsky, Abstract of dis. Doctors Tech. Sciences (1999) [in Russian]

23. O.A. Grebneva, N.N. Novitskii, Thermal Engineering, 61(9), 685 (2014)

24. Yu.P. Adler, E.V. Markova, Yu.V. Granovsky, Planning experiments when searching for optimal conditions (Science, Moscow, 1976) [in Russian]

25. V.V. Fedorov, The theory of optimal experiment (Science, Moscow, 1971) [in Russian]

26. G. A. Korn, T. M. Korn, Mathematical Handbook for Scientists and Engineers (2000)

27. C. Shannon, Works on information theory and cybernetics (1963)

28. N. N. Novitsky, Thesis for the degree of Doctor of Technical Sciences (1999) [in Russian]

29. A.P. Merenkov, V.Ya. Hasilev, Theory of hydraulic circuits (Science, Moscow 1985) [in Russian] 